\title{
An empirical study of Taiwan's food security index
}

\author{
Chih-Yang Yeh ${ }^{1}$, Pei-San Liao ${ }^{1}$, Chieh-Yu Liu ${ }^{2}$, Jeng-Fu Liu ${ }^{1}$ and Hsing-Yi Chang ${ }^{3, *}$ \\ ${ }^{1}$ Graduate Institute of Applied Statistics, Fu Jen Catholic University, Taipei, Taiwan, Republic of China: \\ ${ }^{2}$ Department of Nursing, National Taipei College of Nursing, Taipei, Taiwan, Republic of China: ${ }^{3}$ Center for \\ Health Policy Research and Development, Institute for Population Health Science, National Health Research \\ Institutes, Keyan Road, Zhunan Town, Miaoli County 350, Taiwan, Republic of China
}

Submitted 27 August 2008: Accepted 24 August 2009: First published online 7 October 2009

\begin{abstract}
Objective: The FAO has developed an approach for estimating the prevalence of undernourishment. Based on the FAO method Taiwan has a prevalence of undernourishment of $3.98 \%$, which is higher than that of some developing countries. As this is not a true reflection of the status of undernourishment in our nation, the purpose of the present study was to modify the FAO methodology for Taiwan.

Design: Two factors were considered in the modified version. As the minimum dietary energy requirement was the main factor contributing to the inflated prevalence in Taiwan, we adjusted for a lighter physical activity level, based on the average BMI of the Taiwanese population, and calculated a new minimum dietary energy requirement. We then fitted a second-order polynomial regression model for prediction of per capita dietary energy supply.

Results: The adjusted minimum dietary energy requirement was reduced to $7648 \mathrm{~kJ} / \mathrm{d}$ or $7765 \mathrm{~kJ} / \mathrm{d}$ compared with the original value of $8054 \mathrm{~kJ} / \mathrm{d}$. This resulted in a decrease of the prevalence of undernourishment in Taiwan to $2.5 \%$ or $3 \cdot 0 \%$, which is much closer to that of other countries with the same level of economic development. The second-order polynomial regression model efficiently reduced the variation in dietary energy consumption and resulted in an undernourishment prevalence of less than $2 \cdot 5 \%$.

Conclusions: This new adapted method is more appropriate for Taiwan. It is recommended that each country evaluates the appropriateness of the FAO approach for its population.
\end{abstract}

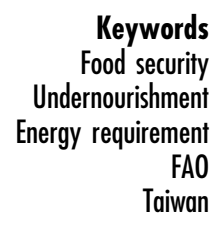

FAO
Continuing environmental degradation has resulted in increasing uncertainty about food supply. Moreover, sustained unequal development and distribution of populations has led to an overabundance of food production in many developed countries alongside long-term food insufficiency in parts of the developing world. There are concerns that the issue of food security could impact on stable global societal development. In order to address the issue of global food security and develop a further understanding of the status of food deprivation in developing countries, the FAO has been estimating the prevalence of undernourishment in connection with the world food survey reports. This report, published every 10 years, uses countries as the unit of estimation and provides global and regional long-term trends in undernourishment.

According to the declaration of the 1996 World Food Summit ${ }^{(1)}$, food security should include the four facets of sufficiency, distribution, stability and sustainability ${ }^{(2)}$. To maintain global food security, not only does each country need to voluntarily agree to set a food security index (which includes measures of food self-sufficiency and food security stockpiles), but it also must take on the responsibility of sharing food resources through regional or global mutual cooperation.

Despite the fact that agricultural development in Taiwan has removed the problem of insufficient food production, following entry into the World Trade Organization, the prices of food commodities in Taiwan follow the rise and fall of the global market. If a situation develops where food imports are insufficient and food reserves are inappropriate, Taiwan has the potential to develop food insecurity. Therefore, stability and sustainability are currently the key food security issues in Taiwan. It is important that Taiwan regularly reports on its food security status to the FAO in order to fulfil its responsibility as a member of global society, to extend the boundaries currently covered by global food status monitoring, and to enable the adoption of appropriate measures when necessary. The purpose of the present study was to investigate the adequacy of the FAO method for estimating the prevalence of undernourishment as an index of Taiwan's food security. 
The FAO has a set of methods for estimating the prevalence of undernourishment in a population ${ }^{(3)}$. This method is based on the relationship between the distribution of household income and per capita food consumption, with the aim of estimating the distribution of per capita food consumption. However, the FAO method assumes that this distribution is influenced by factors associated with unequal distribution of food consumption rather than income-related factors. As a result, it is mainly focused on finding the proportion of those with a food consumption level lower than the minimum dietary energy requirement. There are another two related methods for measuring the prevalence of undernourishment: one provided by the US Department of Agriculture ${ }^{(4)}$ and the other by Senauer and Sur ${ }^{(5)}$. They are similar to the FAO method in that they are based on the relationship between the distribution of household income and per capita food consumption. However, they differ by focusing on investigating which individuals have insufficient incomes that result in them having the lowest level of food consumption.

The FAO method has some drawbacks ${ }^{(5-9)}$ with regard to the accuracy of estimation of the prevalence of undernourishment. Dietary energy consumption has a log-normal distribution; however the FAO method completely relies on three statistics. The first comes from per capita dietary energy supply data from the food balance sheet; the second is the CV of dietary energy consumption, which is predominantly derived from household survey data and is hypothesized to lie between $0 \cdot 2$ and 0.356 ; and the third is the minimum dietary energy requirement that is used to determine the cut-point for undernourishment. We evaluate them one by one in the following sections.

\section{Materials and methods}

\section{Data collection}

A large amount of data was required in order to calculate the prevalence of undernourishment in Taiwan using FAO methodology. This included: the food balance sheet of Taiwan from the Food Supply and Utilization Annual Report by the Council of Agriculture; the Survey of Family Income and Expenditure by the Directorate General of Budget, Accounting and Statistics of Taiwan (DGBAS); data on population age distribution and women of reproductive age from the Department of Statistics, Ministry of the Interior; and the Nutrition and Health Survey in Taiwan (NAHSIT) carried out by the Center for Survey Research, Academia Sinica, Taipei, Taiwan. The application of each of these pieces of data is further described below.

The dietary energy supply component of the FAO method is estimated from the energy supply available per person per day derived from the food balance sheet. It is calculated by multiplying the daily per capita food supply by the food's energy content. The annual survey of family income and expenditure in Taiwan uses households as the sampling frame and is aimed at understanding changes in household income and consumption. The data from this survey used in our study include the number of households, the mean number of members per household, and the cost of food items contributing to consumption expenditure. The household survey data are divided into ten groups evenly based on household income distribution. These are deciles groups of household per capita expenditure. The NAHSIT 1993-1996(10) was carried out from July 1993 to June 1996 throughout Taiwan and investigated the nutrition and health status of Taiwanese people according to sex, age and area. As anthropometric data are required to estimate minimum dietary energy requirement using the FAO method, the major data components used were sex, age, height, weight and BMI.

\section{Definition of variables}

Undernourishment refers to an energy intake that is insufficient for basic requirements. As it is impossible to know definitively the mean per capita daily energy intake and the definition of basic energy requirements will differ from person to person, the FAO method for estimating the prevalence of undernourishment defines basic requirements based on a cut-point on the distribution curve of dietary energy consumption. The proportion of the population with a dietary energy intake below this cutpoint is then estimated. The probability of falling below the cut-point is expressed by the equation ${ }^{(3)}$ :

$$
P(U)=P\left(Y<r_{L}\right)=\int_{y<r_{L}} f(y) \mathrm{d} y=F_{Y}\left(r_{L}\right)
$$

where $P(U)$ is the prevalence of undernourishment, $r_{L}$ is the per capita minimum dietary energy requirement and $y$ is the per capita dietary energy consumption with the distribution $f(y)$, which is assumed to be log-normal ${ }^{(11)}$. As the distribution of dietary energy consumption is skewed to the right, any inaccuracy in estimation of the cut-point will result in large changes to the prevalence of undernourishment. As a result, it is necessary to transform $Y$ to a normal distribution of $X=\ln Y$ and then recalculate $P(U)$. The mean and variance of $X$ can be obtained from the equations:

$$
\mu_{X}=\ln \mu_{Y}-\frac{1}{2} \sigma_{X}^{2}
$$

and

$$
\sigma_{X}^{2}=\ln \left(C V_{Y}^{2}+1\right)
$$

Estimation of $\mu_{X}$ and $\sigma_{X}^{2}$ requires the use of $\mu_{Y}$ and $C V_{Y}$. However, as it is not easy to obtain accurate values of these parameters, the FAO method replaces $\mu_{Y}$ with per capita dietary energy supply. The square root of the sum of the square of the CV of dietary energy supply $C V(y \mid v)$ due to the household per capita income $(v)$ and the square of the $\mathrm{CV}$ of dietary energy requirement $C V(y \mid r)$ 
due to energy requirement $\left(r\right.$ ) can be used in place of $C V_{Y}$ as per the following $(C V(y \mid r)$ is fixed at $0 \cdot 2)$ :

$$
C V_{Y}=\sqrt{C V^{2}(y \mid v)+C V^{2}(y \mid r)}
$$

As we were only able to obtain data on per capita dietary energy supply and did not know the actual variance, the FAO method utilizes the distribution of dietary energy consumption estimated from food commodity consumption expenditure from the household income and expenditure survey ${ }^{(3)}$.

When estimating the minimum dietary energy requirement $r_{L}$ for each country, the FAO method uses the formula:

$$
r_{L}=\sum_{i j}\left(M E R_{i j} \times P_{i j}\right)+P A
$$

where $M E R_{i j}$ is the minimum dietary energy requirement of people of sex $i$ in age group $j ; P_{i j}$ is the proportion of the total national population that belongs to this particular group; and $P A$ or the pregnancy allowance is the energy requirement during pregnancy, which is calculated by multiplying the general fertility rate by $314 \mathrm{~kJ}$. The FAO method uses a different approach for those under 18 years of age and those 18 years of age and above when calculating the minimum energy requirement $M E R_{i j}$. For those under 18 years of age, total energy expenditure (TEE) is obtained from a gender-specific formula that uses the fifth percentile of weight distribution ${ }^{(12)}$ :

$$
\begin{aligned}
& T E E_{\text {Male }}(\mathrm{kJ} / \mathrm{d})=1298+265 \mathrm{Wt}-1 \cdot 1 \mathrm{Wt}^{2} \\
& T E E_{\text {Female }}(\mathrm{kJ} / \mathrm{d})=1102+273 \mathrm{Wt}-1.9 \mathrm{Wt}^{2}
\end{aligned}
$$

where $W t$ refers to weight. Finally, the energy intake required for weight increases associated with growth and development with age are added in ${ }^{(12)}$. For those aged 18 years and over, the weight calculated from the fifth percentile of height distribution and the fifth percentile of BMI is entered into the BMR formula shown in Table $1^{(13)}$. It is then multiplied by the minimum physical activity level (PAL) recommended by the FAO, which is 1.55 and 1.56 in men and women, respectively ${ }^{(3)}$.

As per capita dietary energy consumption $Y$ follows a log-normal distribution, in order to decrease the level of difficulty involved in estimating the prevalence of undernourishment it is necessary for $Y$ to undergo a log transformation of $X=\ln Y$. Then the prevalence of undernourishment can be estimated using the equation:

$$
P(U)=P\left(Y<r_{L}\right)=P\left(X<\ln r_{L}\right)=\Phi\left(\frac{\ln r_{L}-\mu_{X}}{\sigma_{X}}\right)
$$

\section{Statistical analysis}

We used the method recommended by the $\mathrm{FAO}^{(3)}$ to decrease the variance of estimates of $P(U)$ calculated from data from different years. The estimated $\sigma_{X}^{2}$ and $\mu_{X}$ using data from 2002 to 2004 were $0 \cdot 0514$ and $7 \cdot 9596$, respectively. Using the FAO formula, $r_{L}$ was $8054 \mathrm{~kJ}$ and the
Table 1 Equations for estimating BMR

\begin{tabular}{lcc}
\hline Age (years) & BMR $(\mathrm{kJ} / \mathrm{d})$ & SE \\
\hline Men & & \\
$<3$ & $249 W t-127$ & $0 \cdot 292$ \\
$3-9$ & $95 W t+2110$ & $0 \cdot 280$ \\
$10-17$ & $74 W t+2754$ & $0 \cdot 441$ \\
$18-29$ & $63 W t+2896$ & $0 \cdot 641$ \\
$30-59$ & $48 W t+3653$ & $0 \cdot 700$ \\
$\geq 60$ & $49 W t+2459$ & $0 \cdot 686$ \\
Women & & \\
$<3$ & $244 W t-130$ & $0 \cdot 246$ \\
$3-9$ & $85 W t+2033$ & $0 \cdot 292$ \\
$10-17$ & $56 W t+2898$ & $0 \cdot 466$ \\
$18-29$ & $62 W t+2036$ & $0 \cdot 497$ \\
$30-59$ & $34 W t+3538$ & $0 \cdot 465$ \\
$\geq 60$ & $38 W t+2755$ & $0 \cdot 451$ \\
\hline
\end{tabular}

$W t$, weight in kilograms.

Source of data: Schofield (1985) ${ }^{(13)}$

corresponding estimated $P(U)$ was $3.98 \%$. As the average population in Taiwan aged 4 years and above from 2002 to 2004 was about 21.6 million persons, the mean number of persons with undernourishment for these three years was approximately 860000 persons, which might not be the true situation in Taiwan.

Based on NAHSIT 1993-1996 ${ }^{(10)}$, the energy intake estimated from $24 \mathrm{~h}$ recall was $104 \%$ of the Recommend Daily Nutrient Allowance (RDNA) for men aged between 19 and 64 years, whereas that of women was $93 \%$ of the RDNA $^{(14)}$. The within-individual variation was not adjusted for the calculation. Once the within-individual variation is accounted for, the distribution could be narrower than the observed. This means less people would fall in the lower and higher end of the distribution ${ }^{(15)}$. In other words, the energy intakes would be more than enough in the population. Figure 1 shows the time trend of percentage of income spent on different items based on DGBAS. The percentage of income spent on food, beverages and tobacco decreased from about $60 \%$ to less than $25 \%$ between the years 1964 and 2007, whereas the percentage of income spent on recreation, entertainment, education and cultural services increased from about $1 \%$ to $12.5 \%$. During the time of the Asian financial crisis, around 1997-8, the percentage of income spending on these two major items remained stable. This implies that economic growth in the country has enabled people to move from meeting physical needs to meeting psychological needs, and the financial crisis in 1997-8 did not alter that trend. The survey of intakes and the time trend of spending support the fact that the proportion of people in Taiwan suffering from malnutrition was less than that estimated by the original FAO method. Therefore, we need to adjust the FAO method to fit the situation in Taiwan.

As discussed above, the three main parameters influencing the prevalence of undernourishment $P(U)$ are the per capita dietary energy supply $\mu_{Y}$, the $\mathrm{CV}$ of dietary energy consumption $C V_{Y}$ and the minimum dietary energy requirement $r_{L}$. The estimated values of these three 


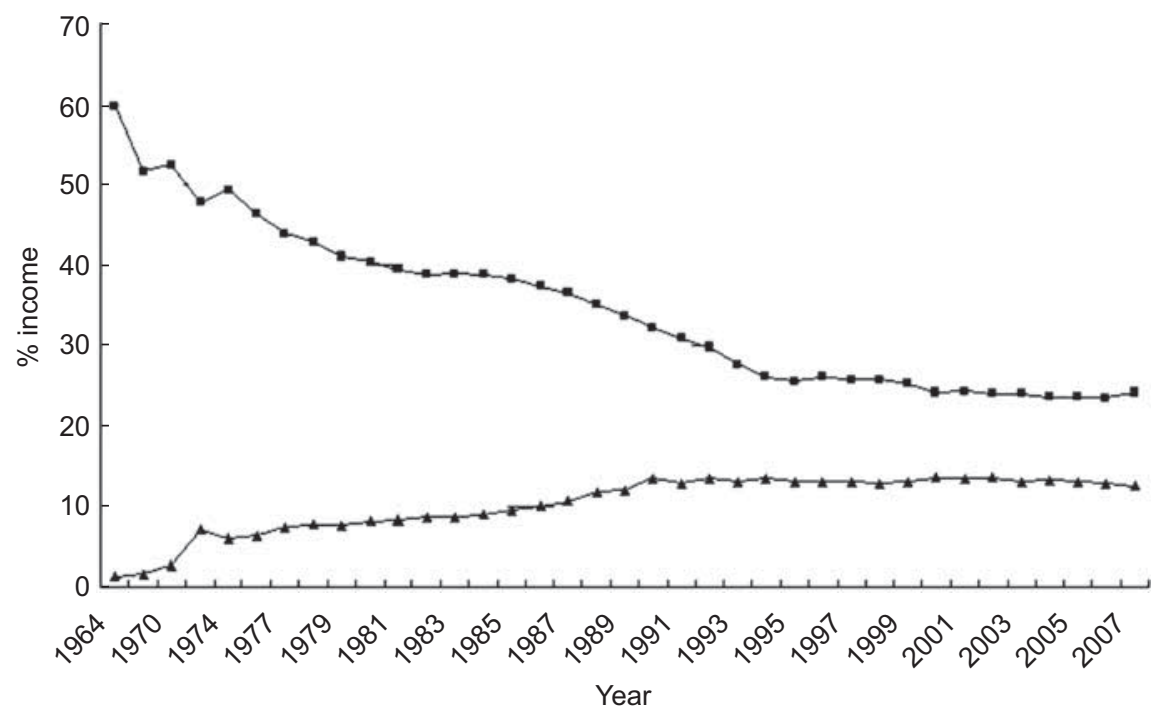

Fig. 1 Time trends of percentage of income spent on food, beverages and tobacco (- - -) and on recreation, entertainment, education and cultural services (- - -) in Taiwan

Table 2 Variation in the prevalence of undernourishment (\%) in Taiwan using the FAO method

\begin{tabular}{lccrr}
\hline & & \multicolumn{2}{c}{ MDER, $r_{L}(\mathrm{~kJ}) / \%$ of original value } \\
\cline { 3 - 5 } Distribution of DEC, $C V_{Y}$ & $\mathrm{DES}, \mu_{Y}(\mathrm{~kJ}) / \%$ of original value & $7248 / 90$ & $8054 / 100$ & $8859 / 110$ \\
\hline 0.20 & $11065 / 90$ & $2 \cdot 1$ & $6 \cdot 6$ & $15 \cdot 3$ \\
& $12294 / 100$ & $0 \cdot 5$ & $2 \cdot 1$ & $6 \cdot 0$ \\
0.23 & $13524 / 110$ & $0 \cdot 1$ & $0 \cdot 6$ & $2 \cdot 1$ \\
& $11065 / 90$ & $4 \cdot 0$ & $9 \cdot 9$ & $19 \cdot 3$ \\
0.26 & $12294 / 100$ & $0 \cdot 4$ & $4 \cdot 0$ & $4 \cdot 2$ \\
& $13524 / 110$ & $6 \cdot 4$ & $13 \cdot 3$ & $22 \cdot 9$ \\
& $11065 / 90$ & $2 \cdot 6$ & $6 \cdot 4$ & $12 \cdot 4$ \\
\hline
\end{tabular}

DEC, dietary energy consumption; DES, dietary energy supply; MDER, minimum dietary energy requirement.

parameters are $12294 \mathrm{~kJ}, 0 \cdot 23$ and $8054 \mathrm{~kJ}$, respectively. We suggest two kinds of corrections to use with the FAO method to improve the accuracy of estimation of the prevalence of undernourishment in Taiwan. Our techniques involve searching for the parameters with the greatest influence on the prevalence estimate, correction of these parameter estimates, and the use of regression analysis to adjust the variance in the dietary energy supply distribution.

\section{Results}

\section{Analysis of parameters associated with the prevalence of undernourishment}

We used Svedberg's ${ }^{(8)}$ method of varying $\mu_{Y}, C V_{Y}$ or $r_{L}$ separately to examine which parameter has a strong influence on the prevalence of undernourishment. Table 2 lists twenty-seven estimates of $P(U)$ calculated $^{(8)}$ with various combinations of $C V_{Y}$ as $0 \cdot 20,0 \cdot 23$ or $0 \cdot 26, \mu_{Y}$ as $11065 \mathrm{~kJ}, 12294 \mathrm{~kJ}$ or $13524 \mathrm{~kJ}$, and $r_{L}$ as $7248 \mathrm{~kJ}, 8054 \mathrm{~kJ}$ or $8859 \mathrm{~kJ}$. The variations of $\mu_{Y}$ and $r_{L}$ are corresponding to $90 \%, 100 \%$ and $110 \%$ of the original values. The estimated prevalence values ranged from $0 \cdot 1 \%$ to $22 \cdot 9 \%$. As shown in Table $2, \mu_{Y}$ is inversely related to $P(U)$, whereas $C V_{Y}$ and $r_{L}$ are positively related to $P(U)$. In addition, as the value of $C V_{Y}$ increases, the magnitude of the influence of $\mu_{Y}$ or $r_{L}$ on change in $P(U)$ also increases. The influence of $r_{L}$ is clearly greater than that of $\mu_{Y}$.

We then widened the range of $C V_{Y}$ and $r_{L}$ and observed the changes closely. For example, if $C V_{Y}$ is fixed at 0.23 and $r_{L}$ is increased or decreased by $50 \%$, the change in $r_{L}$ from $4027 \mathrm{~kJ}$ to $12081 \mathrm{~kJ}$ results in a change in $P(U)$ from about $0 \%$ to $51.4 \%$. When $r_{L}$ is $8859 \mathrm{~kJ}$, the change in the slope is greatest, indicating that if the minimum dietary energy requirement is about $8859 \mathrm{~kJ}$ there will be greater change in the prevalence of undernourishment. Moreover, if there is no change in per capita dietary energy supply and the $\mathrm{CV}$, the prevalence of undernourishment will be most unstable at a minimum energy requirement of $8837 \mathrm{~kJ}$, as shown in Fig. 2.

If $r_{L}$ is fixed at $8104 \mathrm{~kJ}$ and $C V_{Y}$ is increased from $0 \cdot 2$ to $0 \cdot 35$, based on the FAO assumption, $P(U)$ changes from 


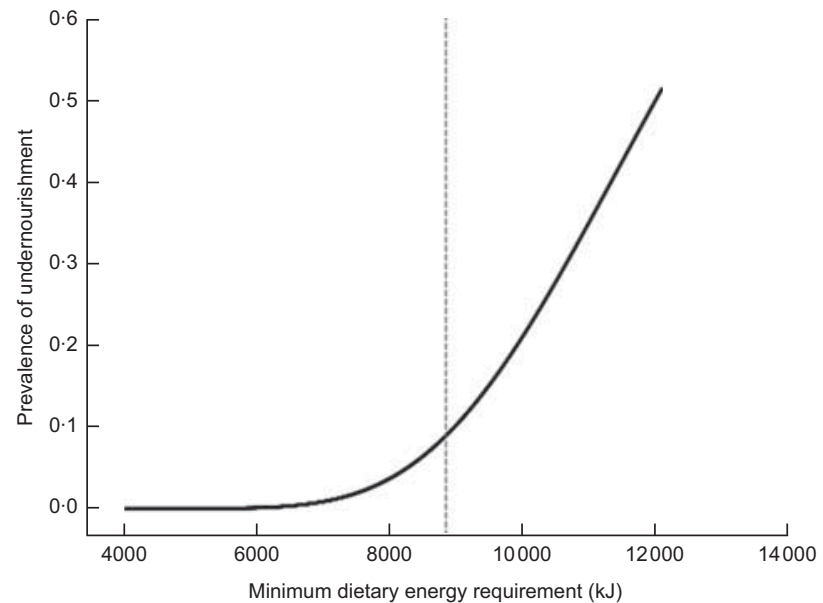

Fig. 2 Variation in prevalence of undernourishment with minimum dietary energy requirement

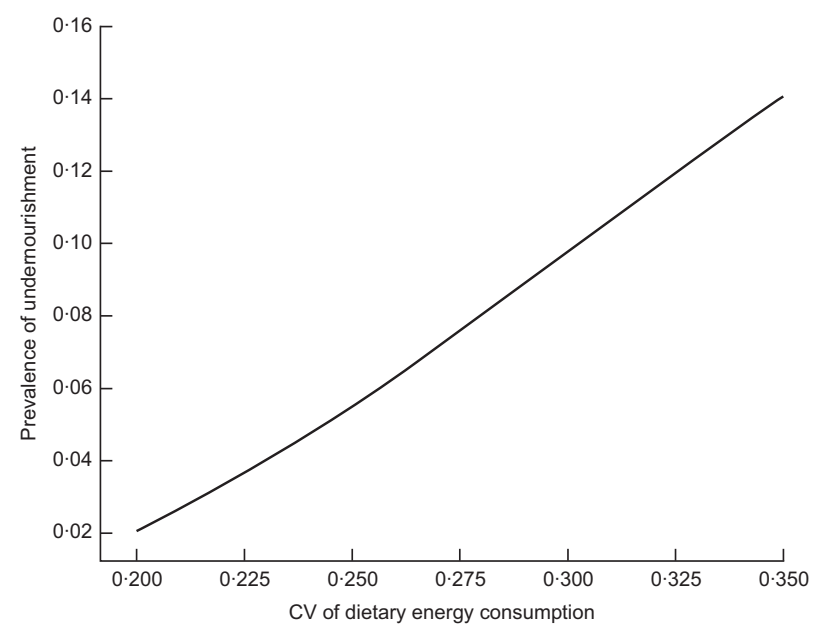

Fig. 3 Variation in prevalence of undernourishment with the CV of dietary energy consumption

$2 \cdot 2 \%$ to $14 \cdot 5 \%$ and the slope does not markedly change. Therefore, change in $C V_{Y}$ leads to little instability in the prevalence of undernourishment as shown in Fig. 3.

From the changes in $P(U)$ shown in detail in Figs 2 and 3 , it can be observed that change in $r_{L}$ has a greater impact on $P(U)$ than change in $C V_{Y}$.

\section{Correction of minimum dietary energy requirement}

As $r_{L}$ has the greatest influence on $P(U)$, based on the FAO equation, its estimation in those aged 18 years and over involves multiplying by the PAL, which may not be appropriate for Taiwanese. We adjusted the value of PAL to improve the estimation of the prevalence of undernourishment in Taiwan.

A PAL of 1.55 means that consumption of $2302 \mathrm{~kJ}$ of energy is required in addition to maintain BMR of $4186 \mathrm{~kJ}$. The main considerations when adjusting PAL are the
Table 3 Comparison of adjustments for lighter physical activity levels in Taiwan, years 2002 to 2004

\begin{tabular}{lcc}
\hline Minimum level of PAL & MDER (kJ) & POU (\%) \\
\hline Males 1.55, females 1.56 & 8054 & 3.98 \\
Adjusted for BMI & 7765 & $2 \cdot 78$ \\
Adjusted using IPAQ Taiwan & 7468 & 1.85 \\
\hline
\end{tabular}

PAL, physical activity level; MDER, minimum dietary energy requirement; POU, prevalence of undernourishment; IPAQ, International Physical Activity Questionnaire.

amount of kilojoules acquired for basic energy requirements and the amount of energy consumed by activity. Therefore, we only need to adjust the 0.55 decimal fraction of the PAL related to activity.

The methodology for estimating the prevalence of undernourishment originally comes from Western countries. Therefore, anthropometric data and recommended PAL values are based on characteristics of Western people and are not suitable for Taiwan. However, as we have no similar local TEE formula, in the present study we were only able to decrease $r_{L}$ by adjusting PAL.

Our first method of adjustment was to adjust the decimal fraction of the PAL recommended by FAO based on the comparison between the mean BMI of Eastern countries (China, Japan, Korea and Singapore) and the mean BMI of Western countries (Canada, the USA, France and the UK). As the BMI of Eastern males is 0.879 times that of Western males, and the BMI of Eastern females is 0.872 times that of Western females, the minimum PAL of Eastern males can be adjusted to 1.483 and the minimum PAL of Eastern females can be adjusted to $1 \cdot 488$.

In the 2001 National Health Interview Survey in Taiwan, about half of the population reported not engaging in any leisure-time physical activities ${ }^{(16)}$. For those who engaged in leisure-time physical activities, less than $20 \%$ reached the $178.5 \mathrm{~kJ}(750 \mathrm{kcal})$ per week that is recommended by WHO. The median energy expenditure was $129 \cdot 5 \mathrm{~kJ}$ ( $544 \mathrm{kcal})$ per week for those who had leisuretime physical activities. According to the International Physical Activity Questionnaire (IPAQ Taiwan) survey conducted by the National Yang-Ming University for the Bureau of Health Promotion ${ }^{(17)}$, $62 \%$ of Taiwanese people were sufficiently physically active. These two observational studies agreed that the energy expenditure of people in Taiwan was low. The mean proportion of those sufficiently active worldwide is $83 \%$, indicating that Taiwan has an activity level of 0.747 times of the global mean. The second method of adjustment involved adjusting the decimal fraction part of the FAO-recommended PAL based on this difference in physical activity level. This resulted in an adjusted PAL of 1.411 for men and 1.418 for women.

The corresponding $r_{L}$ and estimated $P(U)$ for both of the methods of adjustment to PAL are shown in Table 3. Both methods are effective in the estimated $P(U)$ and 
result in an improved estimate of minimum dietary energy requirement in Taiwan.

\section{Distribution of dietary energy consumption}

The FAO method for estimating the prevalence of undernourishment converts food consumption into dietary energy consumption. However, no detailed explanation is provided of the relationship between these two parameters. We used regression analysis to investigate the relationship between food consumption expenditure from the household income survey and dietary energy supply values from the food balance sheet in Taiwan. The corresponding dietary energy supply values were then estimated from mean food consumption expenditure per decile of household per capita expenditure.

Regression analysis was carried out on the household expenditure deciles. In each decile, the mean expenditure from years 1984 to 2001 became the independent variable. The food-balance-sheet-derived dietary energy supply value for the same year became the dependent variable. Selection of models was based on the scatter plot of $Y v . X$, and after careful diagnostics, the model was used to predict the mean value of dietary energy consumption for each decile.

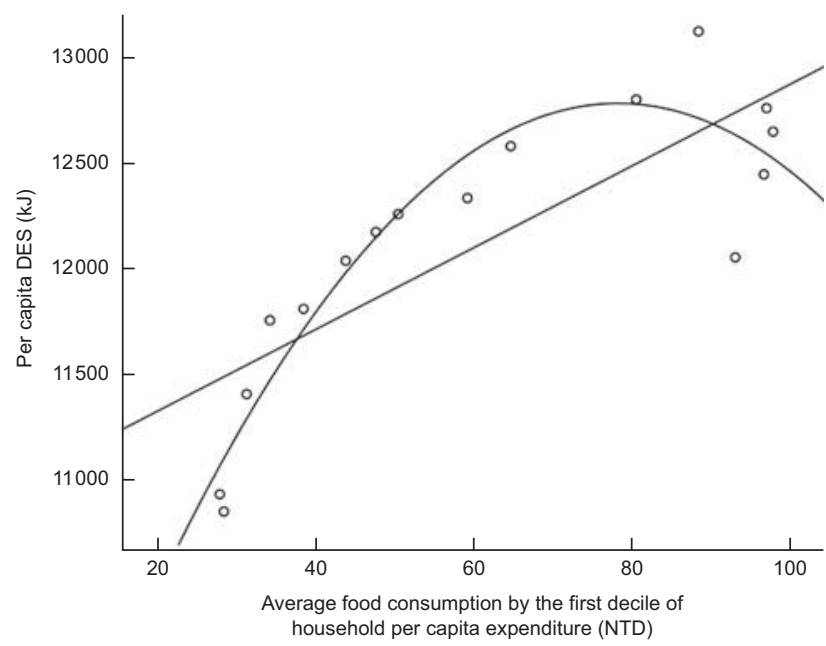

Fig. 4 Scatter plot of per capita dietary energy supply (DES) and average food consumption in the first decile of household per capita expenditure (NTD = new Taiwan dollars)
Taking the first decile of household expenditure as an example, as the scatter plot of $X v . Y$ showed a quadratictype relationship (shown in Fig. 4), we fitted the secondorder polynomial regression model:

$$
Y_{i}=\beta_{0}+\beta_{1} x_{i}+\beta_{11} x_{i}^{2}+\varepsilon_{i}
$$

where $i$ refers to the year and $x$ refers to centralized data $\left(x_{i}=X_{i}-\bar{X}\right)$. The least-squares estimate of the regression function by decile of household expenditure is:

$$
\hat{Y}(\text { decile } 1)=3048 \cdot 905+2 \cdot 658 x-0 \cdot 118 x^{2}
$$

Analysis of residuals found no outliers (there were no studentized residuals of variable observations that had absolute values greater than 3), no violation of independence (Durbin-Watson statistic of 1.478 ), homogeneity of variances ( $P$ value for rank test between the absolute residual value and independent variable was greater than 0.05), normality (Shapiro-Wilk test $P$ value of 0.999 ) and no serious collinearity problems between independent variables ( VIF $=1.528$ ), indicating the goodness-of-fit of the second-order polynomial regression model. In addition, the coefficient of multiple determination $R^{2}$ was over $0 \cdot 872$. Therefore, we believe that the model can be used to predict dietary energy consumption for the first decile of household expenditure.

We used the same method to carry out analyses on the other deciles of expenditure. Fitted models were all second-order polynomial regression models as shown in the second column of Table 4 .

After combining the predicted estimates for the ten deciles of expenditure for 2002 to 2004, a $C V_{Y}$ of about $0 \cdot 20$ was obtained, leading to estimates of $\sigma_{X}^{2}$ and $\mu_{X}$ of 0.039 and $7 \cdot 98$, respectively. Using the original cut-point of $8054 \mathrm{~kJ}$, the corresponding $P(U)$ is $1.75 \%$. However, using the cut-point of $7765 \mathrm{~kJ}$ adjusted for BMI, the corresponding $P(U)$ is $1.09 \%$. Therefore, using the regression analysis results of dietary energy consumption by expenditure deciles leads to a reduced variance in the distribution of dietary energy consumption and a prevalence of undernourishment of under $2 \cdot 5 \%$, which better corresponds to the actual situation in Taiwan. The dietary energy consumption predicted from the second-order

Table 4 Estimates of dietary energy consumption (kJ) in Taiwan in years 2002 to 2004 by decile of household per capita expenditure using a second-order polynomial regression model

\begin{tabular}{rcccccc}
\hline Decile & Equation & SE & 2002 & 2003 & 2004 & Mean \\
\hline 1 & $12762 \cdot 7+11 \cdot 1 x-0 \cdot 5 x^{2}$ & $249 \cdot 09$ & 12567 & 12522 & 12288 & 12549 \\
2 & $12737 \cdot 4+11 \cdot 4 x-0 \cdot 6 x^{2}$ & $272 \cdot 89$ & 12682 & 12571 & 12307 & 12520 \\
3 & $12759 \cdot 8+10 \cdot 7 x-0 \cdot 6 x^{2}$ & $269 \cdot 27$ & 12674 & 12554 & 12269 & 12499 \\
4 & $12780 \cdot 7+10 \cdot 5 x-0 \cdot 6 x^{2}$ & $260 \cdot 75$ & 12683 & 12520 & 12263 & 12489 \\
5 & $12771 \cdot 1+9 \cdot 9 x-0 \cdot 6 x^{2}$ & $261 \cdot 71$ & 12532 & 12540 & 12219 & 12430 \\
6 & $12765 \cdot 3+9 \cdot 9 x-0 \cdot 6 x^{2}$ & $264 \cdot 08$ & 12546 & 12506 & 12260 & 12437 \\
7 & $12775 \cdot 6+9 \cdot 9 x-0 \cdot 5 x^{2}$ & $259 \cdot 61$ & 12556 & 12580 & 12296 \\
8 & $12805 \cdot 8+9 \cdot 0 x-0 \cdot 5 x^{2}$ & $246 \cdot 52$ & 12554 & 12543 & 12266 & 12477 \\
9 & $12811 \cdot 8+8 \cdot 8 x-0 \cdot 5 x^{2}$ & $245 \cdot 95$ & 12545 & 12529 & 12311 & 12461 \\
10 & $12835 \cdot 5+8 \cdot 3 x-0 \cdot 4 x^{2}$ & $254 \cdot 74$ & 12616 & 12545 & 12319 & 12493 \\
\hline
\end{tabular}


polynomial regression model can be used to estimate the prevalence of undernourishment, which can be used as the food security index for Taiwan. A widening gap between rich and poor will show itself in food consumption expenditure, leading to greater variance in dietary energy consumption obtained through the regression function. This in turn will result in a clear increasing trend in the prevalence of undernourishment, enabling Taiwan to determine the presence of unequal food distribution in a timely manner.

\section{Discussion}

The present study modified the FAO method to estimate the proportion of undernourishment in Taiwan. We first modified the minimum dietary energy requirement based on the BMI distribution and PAL of people in Taiwan. The second modification was to fit second-order polynomial regression equations to estimate per capita dietary energy supply. The modifications resulted in reasonable results for Taiwan.

Our study found that the major influential factor on the prevalence of undernourishment was minimum dietary energy requirement. The minimum energy requirement of $8054 \mathrm{~kJ}$ estimated in the original FAO method appears high for the dietary habits of Taiwanese people, and does not appropriately represent per capita daily minimum dietary energy requirements in Taiwan. Therefore, when estimating minimum dietary energy requirement it is necessary to adjust PAL based on the methods in our study involving BMI or physical activity. This new adjusted PAL value results in a minimum dietary energy requirement value that is closer to the actual situation in Taiwan. The improved accuracy of this estimate would be of benefit for FAO monitoring of regional food security.

The FAO method does not provide a detailed explanation of how food consumption expenditure is transformed into the distribution of dietary energy consumption. Even though the $\mathrm{CV}$ of dietary energy consumption is not the major factor influencing the prevalence of undernourishment, we believe that this relationship is worth describing adequately. We used regression analysis to show the distribution of dietary energy consumption for the ten deciles of household per capita expenditure and then calculated the corresponding CV. Our results showed that our adjustments effectively reduced the prevalence of undernourishment in Taiwan from a rate of 40 persons per 1000 to 17 persons per 1000 . That is close to the real situation.

For the purpose of timely monitoring of global undernourishment, Taiwan is considered to be part of the Eastern Asia region which includes China, North and South Korea and Mongolia, based on the global regional demarcations in the most recent FAO statistics ${ }^{(18)}$ and State of Food Insecurity (SOFI) report. From 2002 to 2004 the prevalence of undernourishment in this region was $12 \%$, which equates to an undernourished population of 1.629 billion people. This indicates that the severity of undernourishment in this region is of a medium level ${ }^{(19)}$. As statistics for China include the special administrative regions of Hong Kong and Macao and the province of Taiwan, on the world hunger map drawn up by the FAO, data for Taiwan are the same as those for China. As well as understanding the status of food insecurity in the region, regional monitoring should also focus on any regional inequalities in food distribution. This would help to facilitate mutual assistance between countries aimed at solving regional food security issues. However, in this instance data from Taiwan have been omitted by the FAO, which would be a potential hazard for the FAO's goal to eliminate the population experiencing food deprivation by the year 2015. Table 5 shows the dietary energy consumption, minimum dietary energy requirement and prevalence of undernourishment for the East Asian region according to SOFI. As North Korea and Mongolia are countries of more northern latitudes, they require more dietary energy consumption. The data clearly show that this consumption is insufficient and as a result the prevalence of undernourishment is high in these countries. On the other hand, Taiwan is located at lower latitude and therefore the minimum dietary energy requirement should be lower than that of the other four countries. Our final model provides a reasonable estimate.

Using the FAO method for estimating the prevalence of undernourishment, the main limitation of our study is that the distribution of dietary energy consumption in Taiwan

Table 5 Dietary energy consumption (DEC), minimum dietary energy requirement (MDER) and prevalence of undernourishment (POU) for the East Asian region according to FAO statistics (2002 to 2004) and the State of Food Insecurity Report

\begin{tabular}{lccc}
\hline Country & DEC $(\mathrm{kJ})$ & MDER $(\mathrm{kJ})$ & POU $(\%)$ \\
\hline China & 12265 & - & 12 \\
North Korea & 9125 & 7953 & 33 \\
South Korea & 12684 & 8079 & $<2 \cdot 5$ \\
Mongolia & 9419 & 7828 & 27 \\
Taiwan: original FAO method & 12294 & 8054 & $3 \cdot 98$ \\
Taiwan: first method of adjustment & 12294 & 7765 & $2 \cdot 78$ \\
Taiwan: second method of adjustment & 12294 & 7468 & $1 \cdot 85$ \\
\hline
\end{tabular}

- indicates no data. 
cannot be measured regularly. Moreover, when estimating the minimum dietary energy requirement, there is a lack of applicable equations for estimation of BMR and TEE for Taiwan. As a result, we could only follow the basic essence of the FAO method with our limited data to provide the international community with information about Taiwan.

\section{Acknowledgements}

Source of funding: Part of the study was sponsored by the Council of Agriculture. Conflicts of interest: None. Author contributions: C.-Y.Y. analysed and drafted the manuscript. P.-S.L. advised C.-Y.Y. on the research. She provided insightful discussions on the topic and revised the manuscript many times. C.-Y.L. and J.-F.L. were the committee members who provided intensive discussions on the subject. H.-Y.C. initiated and supervised the whole research. Acknowledgements: We would like to thank the Center for Survey Research, Academia Sinica for providing us with the database from the NAHSIT; the Council of Agriculture for the food balance sheet from the Food Supply and Utilization Annual Report; and the DGBAS for providing the data from the Survey of Family Income and Expenditure.

\section{References}

1. Food and Agriculture Organization of the United Nations (1996) Rome Declaration on World Food Security and World Food Summit Plan of Action. http://www.fao.org/ docrep/003/w3613e/w3613e00.HTM (accessed September 2009).

2. Woo RJ (1998) Adjustment of food security policy for joining the WTO. Taiwan Econ 264, 3-13.

3. Food and Agriculture Organization of the United Nations (2003) FAO Methodology for the Measurement of Food Deprivation. http://www.deart.unifi.it/romano/ASP/FAO $\% 20$ undernourishment $\% 20$ methodology.pdf (accessed September 2009).

4. Shapouri S \& Rosen S (coordinator) (2000) International Agricultural and Trade Reports, Food Security Assessment,
Situation and Outlook Series, GFA-12. Washington, DC: US Department of Agriculture, Economic Research Service.

5. Senauer B \& Sur M (2001) Ending global hunger in the 21st century: projections of the number of food insecure people. Rev Agric Econ 23, 68-81.

6. Gabbert S \& Weikard H-P (2001) How widespread is undernourishment? A critique of measurement methods and new empirical results. Food Policy 26, 209-228.

7. Hayter JE \& Henry CJ (1994) A re-examination of basal metabolic rate predictive equations: the importance of geographical origin of subjects in sample selection. Eur J Clin Nutr 48, 702-707.

8. Svedberg P (1999) 841 million undernourished? World Dev 7, 2081-2098.

9. Svedberg P (2002) Undernutrition overestimated. Econ Dev Cult Change 51, 5-36.

10. Pan WH, Kao MD, Tzeng MS et al. (1999) Nutrition and Health Survey in Taiwan (NAHSIT) 1993-1996: design, contents, and operations. Nutr Sci J 24, 1-10.

11. Food and Agriculture Organization of the United Nations (1987) The Fifth World Food Survey. Rome: FAO.

12. Tontisirin K \& de Haen H (2001) Human Energy Requirements. Report of a Joint FAO/WHO/UNU Expert Consultation, Rome, 17-24 October 2001. FAO Food and Nutrition Technical Report Series no. 1. Rome: FAO.

13. Schofield WN (1985) Predicting basal metabolic rate, new standards and review of previous work. Hum Nutr Clin Nutr 39, Suppl. 1, S5-S41.

14. Pan W-H, Chang Y-H, Chen J-Y et al. (1999) Nutrition and Health Survey in Taiwan (NAHSIT) 1993-1996: dietary nutrient intakes assessed by 24-hour recall. Nutr Sci J 24, 11-39.

15. Chang H-Y, Suchindran CM \& Pan W-H (1999) Using the overdispersed exponential family to estimate the distribution of usual daily intakes of people aged between 18 and 28 in Taiwan. Stat Med 20, 2337-2350.

16. Wei JPM, Wen CP, Chan H-T et al. (2008) Assessing physical activity in an Asian country: low energy expenditure and exercise frequency among adults in Taiwan. Asia Pac J Clin Nutr 17, 297-308.

17. Liou YM, Hung YT, Chiang LC et al. (2006) International Physical Activity Questionnaire Taiwan. Yang-Ming, ROC: National Yang-Ming University \& Bureau of Health Promotion.

18. Food and Agriculture Organization of the United Nations (2006) FAOSTAT. http://www.fao.org/economic/ess/foodsecurity-statistics/en/ (accessed October 2006).

19. Sibrián R, Ramasawmy S \& Mernies J (2006) Measuring Hunger at Sub National Levels from Housebold Surveys Using the FAO Approach: Manual. Rome: FAO. 